\title{
Adjuvant and neoadjuvant treatment of melanoma
}

\author{
Peter Koelblinger (D)
}

Received: 31 January 2020 / Accepted: 25 March 2020 / Published online: 15 April 2020

(C) The Author(s) 2020

\begin{abstract}
Summary For years, interferon alpha was the sole option in the adjuvant treatment of patients with completely resected melanoma with lymph node metastases and a high risk of disease recurrence, albeit being associated with a relatively low efficacy combined with significant toxicities. After the advent of immunotherapy and targeted therapy in locally advanced or metastatic melanoma at the beginning of the last decade, these therapeutic approaches have meanwhile also shown superior efficacy compared to previously used treatments or observation in the context of adjuvant therapy. Hence, adjuvant targeted or anti-PD1-antibody-based immunotherapy was incorporated into routine clinical practice to reduce the risk of tumor recurrence in affected patients in early 2018. Moreover, modern melanoma therapies are increasingly being investigated in a neoadjuvant setting in analogy to other solid malignancies. Considering the promising results reported so far, neoadjuvant immunotherapy might potentially become the treatment of choice in high-risk melanoma patients with macrometastatic disease in the near future.
\end{abstract}

Keywords Melanoma - Adjuvant · Neoadjuvant · Targeted therapy $\cdot$ PD1 antibody

\section{Introduction}

The emergence of targeted therapy and immunotherapy has significantly changed the treatment landscape of locally advanced or metastatic melanoma during the last decade. Unprecedented 5-year survival rates of more than $50 \%$ after combined immunotherapy

P. Koelblinger, M.D. ( $\varangle)$

Department of Dermatology and Allergology, Paracelsus

Medical University, Müllner Hauptstraße 48, 5020 Salzburg, Austria

p.koelblinger@salk.at with ipilimumab and nivolumab in advanced stage melanoma are promising for affected patients, particularly when compared to historic data [1]. Nevertheless, melanoma-associated mortality remains significant. Hence, increasing efforts have been made to investigate the use of modern melanoma therapeutics earlier in the course of the disease, aiming to prevent tumor recurrence and development of metastases. In early 2018, this led to the approval of both targeted therapy and PD1 (programmed death 1)-antibody-based immunotherapy in the adjuvant treatment setting. The present review will summarize the pivotal studies published to date in this context and also focus on the next development in early medical treatment of melanoma-the neoadjuvant approach.

\section{Adjuvant targeted therapy}

The Combi-AD trial investigated the combination of the BRAF inhibitor dabrafenib (75 mg twice daily) and the MEK inhibitor trametinib (2 mg once daily, D+T) compared to placebo in patients with completely resected, BRAF V600 E or K mutated American Joint Committee on Cancer (AJCC) stage III melanoma with lymph node metastases [2]. In this double blind, randomized trial, 870 patients received treatment with $\mathrm{D}+\mathrm{T}$ or placebo for a total of 12 months or until the occurrence of disease recurrence, death or unacceptable toxicities. First results of this trial were presented in late 2017 [2]. In 2018, after a median follow-up of 44 months in the D+T arm, an updated analysis of recurrence-free survival (RFS) - which was the primary outcome of this trial-was published [3]. The 3- and 4 -year RFS rates were 59 and $54 \%$ in the D+T arm and 40 and $38 \%$ in the placebo arm, respectively, resulting in a hazard ratio (HR) of 0.49 (95\% confidence interval [CI] 0.40-0.59). The differences in distant metastasisfree (DMFS) and overall survival (OS) were also signif- 
icant in this trial with HRs of 0.53 (95\% CI 0.42-0.67) and 0.57 (95\% CI 0.42-0.79), respectively. Based on these data, an estimated cure rate of $54 \%$ was calculated for treatment with $\mathrm{D}+\mathrm{T}$ compared with $37 \%$ for placebo. The toxicity profile and tolerability of $\mathrm{D}+\mathrm{T}$ in the combi-AD trial was comparable to previous results in advanced melanoma [4]. Only the $26 \%$ rate of treatment discontinuations was increased compared to what is known from the metastatic setting. This was primarily attributed to the nature of adjuvant treatment per se.

The BRIM8 trial was the only randomized, doubleblind, placebo-controlled trial comparing BRAF inhibitor monotherapy (with vemurafenib) with placebo in 498 patients with stage IIC to IIIC melanoma [5]. The study failed to meet its primary endpoint of improved disease-free survival (DFS) in the second cohort of the trial, which only included patients with stage IIIC disease (median DFS 23.1 versus 15.4 months [HR $0.80,95 \%$ CI $0.54-1.18 ; p$-value: $0.26]$ ). In cohort 1 of the trial (AJCC stage IIC, IIIA and IIIB), DFS outcome was in favor of vemurafenib with a HR of 0.54 (95\% CI 0.37-0.78; $p$-value: 0.001 ) suggesting an exclusive effect of adjuvant BRAF inhibitor monotherapy in patients with lower tumor burden before surgery. The clinical relevance of this finding is limited, as combined BRAF and MEK inhibitor treatment has evolved as standard of care if targeted therapy is considered in an adjuvant treatment situation [6].

\section{Adjuvant immunotherapy}

From 1999 until 2018, interferon alpha (IFN) was the only approved substance for adjuvant treatment of patients with completely resected melanoma in Europe. Although the therapeutic effect of IFN was modest and most likely limited to patients with ulcerated primary tumors [7], large meta-analyses including several thousands of patients demonstrated a statistically significant improvement of both RFS and OS in patients treated with IFN [8, 9] However, this therapeutic effect was relatively small (HR range for recurrence versus observation: $0.82-0.86$, for death: 0.89-0.90), while toxicity of the different IFN schemes is generally significant.

Most recently, results of a double-blind randomized trial comparing high dose IFN with two different doses $(3 \mathrm{mg} / \mathrm{kg}$ and $10 \mathrm{mg} / \mathrm{kg}$ ) of the first "modern" immunotherapy-the cytotoxic T-lymphocyteassociated protein 4 (CTLA-4) antibody ipilimumab (ipi)—were published [10]. Interestingly, efficacy of the two ipilimumab dose regimens investigated in this trial was similar, while toxicity was significantly higher with the $10 \mathrm{mg} / \mathrm{kg}$ dose (rate of treatmentrelated adverse events (AEs) CTC grade 3 and higher: 57 vs. 38\%). This is in contrast to findings in the metastatic setting showing improved efficacy with higher doses of ipilimumab [11]. Compared to adju- vant IFN, ipi $3 \mathrm{mg} / \mathrm{kg}$ was shown to significantly improve OS (HR 0.78; 95.6\% CI 0.61-0.99; $p$-value: 0.044 ), and achieved a trend towards improved RFS ( $p$-value: 0.065) [10]. In light of the effective new treatment options presented within this article, IFN is increasingly considered outdated as an adjuvant treatment option for melanoma. However, it currently remains the only substance which has also been investigated in patients with AJCC stage II melanoma and its use appears to remain justified in this subgroup of patients, particularly in those with an ulcerated primary tumor.

Adjuvant ipilimumab therapy was compared with observation in the placebo-controlled EORTC 18071 trial in AJCC stage III melanoma [12-14]. In this phase III trial, patients treated with ipi did not receive the $3 \mathrm{mg} / \mathrm{kg}$ dose approved in the metastatic setting, but an increased dose of $10 \mathrm{mg} / \mathrm{kg}$. Although both a significant improvement in RFS (HR 0.75, 95\% CI 0.63-0.88) and OS (HR 0.73, 95\% CI 0.60-0.89) were demonstrated for patients treated with adjuvant ipi, the results of this trial only led to approval of adjuvant ipilimumab in the United States, but not in Europe. This was possibly related to the substantial rate of severe (Common Toxicity Criteria [CTC] grade 3 and higher) treatment-related adverse events of $54 \%$ with this regimen including AEs with lethal outcome in approximately $1 \%$ of patients. Regardless of approval status, the importance of ipilimumab in the adjuvant treatment of melanoma rapidly diminished, once the results of the following study on adjuvant PD1antibody treatment were presented in late 2017.

The CheckMate 238 trial enrolled 906 patients with completely resected AJCC stage IIIB, IIIC or IV melanoma who were randomized in a 1:1 fashion to receive either $3 \mathrm{mg} / \mathrm{kg}$ of the anti-PD1-antibody nivolumab (nivo) every 2 weeks for up to 1 year or $10 \mathrm{mg} / \mathrm{kg}$ of ipilimumab every 3 weeks for 4 doses, followed by maintenance treatment every 12 weeks [15]. The 3-year follow-up results of this trial were recently presented [16]. Adjuvant nivo continued to be superior to ipi in terms of the primary endpoint RFS. Including all tumor stages, 3-year RFS rates were $58 \%$ with nivo versus $45 \%$ with ipi, resulting in a HR of 0.68 (95\% CI 0.56-0.82). DMFS was also superior with nivolumab in patients with stage III disease (HR $0.78,95 \%$ CI $0.62-0.99$ ). Due to the limited follow-up, OS data of this trial have not been presented to date. Yet, based on the superior RFS data, nivolumab was the first anti-PD1-antibody to be approved for the adjuvant treatment of completely resected stage III melanoma with lymph node metastases and also for resected stage IV melanoma.

The second anti-PD1-antibody that has been approved for the adjuvant treatment of melanoma is pembrolizumab (pembro). This approval was based on the results of the KEYNOTE-054 trial which investigated adjuvant pembro (200 mg flat dose every 3 weeks) compared with placebo [17]. In contrast to 
the CheckMate238 trial with nivolumab, this trial also included patients with stage IIIA melanoma, but no patients with stage IV disease. The primary endpoint of improved RFS in the overall patient population was reached in the first analysis of this trial after a median follow-up of 15 months. The 1-year RFS rates were $75.4 \%$ in the pembro and $61 \%$ in the placebo arm resulting in a HR for recurrence or death of $0.57(98.4 \%$ CI 0.43-0.74).

Within the pivotal adjuvant trials outlined above, both anti-PD1 inhibitors were well tolerated with a discontinuation rate due to AEs of $10 \%$ with nivo and $14 \%$ with pembro $[15,17]$. Treatment-related AEs of CTC grade 3 and higher occurred in approximately $15 \%$ of patients in both trials. However, in contrast to AEs with targeted therapy, certain immune-related AEs can result in long-term consequences for affected patients (e.g., hypophysitis, diabetes) or can be potentially life threatening with lethal outcome in rare cases. This should particularly be taken into account when counselling patients with BRAF-mutated melanoma-in which there is an alternative treatment option-or those with a relatively low risk of recurrence about adjuvant immunotherapy. A potential biomarker that may facilitate decision making when choosing between adjuvant targeted and immunotherapy in BRAF-mutated melanoma is tumor mutational burden (TMB). In an exploratory analysis of the Combi-AD trial, Dummer et al. showed that ongoing RFS benefit with $\mathrm{D}+\mathrm{T}$ treatment is limited to patients with low TMB, which applied to approximately two thirds of patients in this trial [18]. The HRs comparing $\mathrm{D}+\mathrm{T}$ versus placebo in patients with low and high TMB were 0.49 (95\% CI 0.35-0.68) and 0.75 (95\% CI 0.44-1.26). Evaluation of interferon gamma gene expression could add additional prognostic information in this context. Prospective confirmation of these findings is necessary before incorporation into routine clinical practice.

\section{Neoadjuvant treatment}

Since adjuvant targeted and immunotherapy were implemented into routine clinical practice in early 2018, the next hot topic in early melanoma treatment has been the development of neoadjuvant treatment approaches. This strategy is currently limited to the relatively small subgroup of patients who present with macrometastatic lymph node disease (AJCC stage IIIB-D). Nevertheless, the prognostic and therapeutic potential of neoadjuvant treatment is appealing. This has even led to the formation of an International Neoadjuvant Melanoma Consortium which has recently published consensus-based recommendations on neoadjuvant systemic therapy [19]. Treatment approaches with both targeted (BRAF/MEK inhibitor combination) and immunotherapy have been investigated in the neoadjuvant setting [20-25]. The treatment duration before surgery typically ranged from 3 to 12 weeks in these trials, frequently followed by a period of adjuvant treatment resulting in a total treatment duration of 1 year. A pooled analysis of six relevant neoadjuvant trials including a total of 184 patients who eventually underwent surgery after neoadjuvant induction therapy showed that the rate of complete pathological responses (pCRs) appears to be comparable (around 50\%) after combined targeted and combined immunotherapy (ipi + nivo) [26]. However, follow-up data of this analysis depicted favorable RFS outcomes in patients treated with neoadjuvant immunotherapy. For example, after a median followup of 10 months, in 51 patients who achieved a pCR after neoadjuvant immunotherapy, not a single recurrence event had occurred. As toxicity is a particular issue with neoadjuvant combined immunotherapy, different dosing schedules have been compared in the largest neoadjuvant trial conducted to date (OpACINneo) [25]. A regimen with two cycles of ipi $1 \mathrm{mg} / \mathrm{kg}$ plus nivo $3 \mathrm{mg} / \mathrm{kg}$ before surgery without subsequent adjuvant therapy appeared to be most suitable for use in future neoadjuvant trials. This regimen was associated with a pathological response rate of $77 \%$ (57\% pCRs) and a grade $3 / 4$ AE rate of $20 \%$ within the first 12 weeks of treatment.

\section{Summary and future outlook}

The approval of novel adjuvant treatments has significantly changed the clinical management of patients with completely resected stage III (and IV) melanoma in recent years. Both adjuvant targeted therapy and PD1-antibody-based immunotherapy are able to relatively reduce the risk of recurrence by approximately $50 \%$. As efficacy of targeted and immunotherapy appears to be similar in patients with a BRAF mutation, the decision for one or the other treatment remains to be difficult for both physicians and patients. Accordingly, current melanoma treatment guidelines equally recommend either adjuvant targeted or immunotherapy for patients with BRAF-mutated stage IIIA-D melanoma, mentioning the differences in frequency and severity of AEs discussed above [6]. Although the use of adjuvant treatment can be questioned critically in low-risk populations such as patients with stage IIIA disease [27], ongoing studies are also investigating adjuvant anti-PD1-therapy in patients with thick primary melanomas without lymph node involvement (AJCC stage IIB and IIC, see [28] and ClinicalTrials.gov Identifier: NCT04099251).

In patients with macrometastatic lymph node disease-which is associated with a considerably poorer prognosis - the promising neoadjuvant treatment approach will be further examined and could potentially be integrated into routine clinical practice in the near future. 


\section{Take home message}

PD1 antibodies or combined BRAF- and MEK-inhibition are considered as the current standard of care in adjuvant treatment of completely resected stage III (and IV) melanoma.

Funding Open access funding provided by Paracelsus Medical University.

Conflict of interest P. Koelblinger has received honoraria for travel support and consulting/advisory roles from Roche, Bristol Myers Squibb (BMS), Merck Sharp \& Dome (MSD), Novartis, Pierre Fabre, Sanofi Aventis and Amgen outside the submitted work.

Open Access This article is licensed under a Creative Commons Attribution 4.0 International License, which permits use, sharing, adaptation, distribution and reproduction in any medium or format, as long as you give appropriate credit to the original author(s) and the source, provide a link to the Creative Commons licence, and indicate if changes were made. The images or other third party material in this article are included in the article's Creative Commons licence, unless indicated otherwise in a credit line to the material. If material is not included in the article's Creative Commons licence and your intended use is not permitted by statutory regulation or exceeds the permitted use, you will need to obtain permission directly from the copyright holder. To view a copy of this licence, visit http://creativecommons.org/licenses/by/4.0/.

\section{References}

1. Larkin J, Chiarion-Sileni V, Gonzalez R, Grob JJ, Rutkowski P, Lao CD, et al. Five-year survival with combined nivolumab and Ipilimumab in advanced melanoma. $\mathrm{N}$ Engl J Med. 2019;381(16):1535-46. https://doi.org/10.1056/ NEJMoa1910836.

2. Long GV, Hauschild A, Santinami M, Atkinson V, Mandala M, Chiarion-Sileni V, et al. Adjuvant dabrafenib plus trametinib in stage III BRAF-mutated melanoma. N Engl J Med. 2017;377(19):1813-23. https://doi.org/10.1056/ NEJMoa1708539.

3. Hauschild A, Dummer R, Schadendorf D, Santinami M, Atkinson V, Mandala M, et al. Longer follow-up confirms relapse-free survival benefit with adjuvant dabrafenib plus trametinib in patients with Resected BRAF V600-mutant stage III melanoma. J Clin Oncol. 2018; https:// doi.org/10. 1200/JCO.18.01219.

4. Long GV, Eroglu Z, Infante J, Patel S, Daud A, Johnson $\mathrm{DB}$, et al. Long-term outcomes in patients with BRAF V600-mutant metastatic melanoma who received dabrafenib combined with trametinib. J Clin Oncol. 2017; https://doi.org/10.1200/jco.2017.74.1025.

5. Maio M, Lewis K, Demidov L, Mandala M, Bondarenko I, Ascierto PA, et al. Adjuvant vemurafenib in resected, BRAF(V600) mutation-positive melanoma (BRIM8): a randomised, double-blind, placebo-controlled, multicentre, phase 3 trial. Lancet Oncol. 2018;19(4):510-20. https:// doi. org/10.1016/S1470-2045(18)30106-2.

6. Garbe C, Amaral T, Peris K, Hauschild A, Arenberger P, Bastholt L, et al. European consensus-based interdisciplinary guideline for melanoma. Part 2: Treatment-Update 2019. Eur J Cancer. 2019; https://doi. org/10.1016/j.ejca.2019.11.015.
7. Eggermont AM, Suciu S, Rutkowski P, Kruit WH, Punt CJ, Dummer R, et al. Long term follow up of the EORTC 18952 trial of adjuvant therapy in resected stage IIB-III cutaneous melanoma patients comparing intermediate doses of interferon-alpha-2b (IFN) with observation: Ulceration of primary is key determinant for IFN-sensitivity. Eur J Cancer. 2016;55:111-21. https://doi.org/10.1016/j.ejca.2015. 11.014 .

8. Ives NJ, Suciu S, Eggermont AMM, Kirkwood J, Lorigan P, Markovic SN, et al. Adjuvant interferon-alpha for the treatment of high-risk melanoma: an individual patient data meta-analysis. Eur J Cancer. 2017;82:171-83. https:// doi.org/10.1016/j.ejca.2017.06.006.

9. Mocellin S, Pasquali S, Rossi CR, Nitti D. Interferon alpha adjuvant therapy in patients with high-risk melanoma: a systematic review and meta-analysis. J Natl Cancer Inst. 2010;102(7):493-501. https://doi.org/10.1093/jnci/ djq009.

10. Tarhini AA, Lee SJ, Hodi FS, Rao UNM, Cohen GI, Hamid O, et al. Phase III study of Adjuvant Ipilimumab (3 or $10 \mathrm{mg} / \mathrm{kg}$ ) versus high-dose interferon alfa- $2 b$ for Resected high-risk melanoma: north American intergroup E1609. JClin Oncol. 2019; https://doi.org/10.1200/JCO.19.01381.

11. Ascierto PA, Del Vecchio M, Robert C, Mackiewicz A, Chiarion-Sileni V, Arance A, et al. Ipilimumab $10 \mathrm{mg} / \mathrm{kg}$ versus ipilimumab $3 \mathrm{mg} / \mathrm{kg}$ in patients with unresectable or metastatic melanoma: a randomised, double-blind, multicentre, phase 3 trial. Lancet Oncol. 2017;18(5):611-22. https://doi.org/10.1016/S1470-2045(17)30231-0.

12. Eggermont AM, Chiarion-Sileni V, Grob JJ, Dummer R, Wolchok JD, Schmidt H, et al. Prolonged survival in stage III melanoma with ipilimumab adjuvant therapy. N Engl J Med. 2016;375(19):1845-55. https://doi.org/10.1056/ NEJMoa1611299.

13. Eggermont AM, Chiarion-Sileni V, Grob JJ, Dummer R, Wolchok JD, Schmidt H, et al. Adjuvant ipilimumab versus placebo after complete resection of high-risk stage III melanoma (EORTC 18071): a randomised, double-blind, phase 3 trial. Lancet Oncol. 2015;16(5):522-30. https://doi. org/10.1016/S1470-2045(15)70122-1.

14. Eggermont AMM, Chiarion-Sileni V, Grob JJ, Dummer R, Wolchok JD, Schmidt H, et al. Adjuvant ipilimumab versus placebo after complete resection of stage III melanoma: long-term follow-up results of the European Organisation for Research and Treatment of Cancer 18071 double-blind phase 3 randomised trial. Eur J Cancer. 2019;119:1-10. https://doi.org/10.1016/j.ejca.2019.07.001.

15. Weber J, Mandala M, Del Vecchio M, Gogas HJ, Arance AM, Cowey CL, et al. Adjuvant nivolumab versus Ipilimumab in resected stage III or IV melanoma. N Engl J Med. 2017; https://doi.org/10.1056/NEJMoa1709030.

16. Weber J, Del Vecchio M, Mandala M, Gogas H, Arance A, Dalle S, et al. Adjuvant nivolumab (NIVO) versus ipilimumab (IPI) in resected stage III/IV melanoma: 3-year efficacy and biomarker results from the phase III CheckMate 238 trial. conference abstract. Oxford: Oxford University Press; 2019. pp. 533-4.

17. Eggermont AMM, Blank CU, Mandala M, Long GV, Atkinson V, Dalle S, et al. Adjuvant pembrolizumab versus placebo in resected stage III melanoma. N Engl J Med. 2018;378(19):1789-801. https://doi.org/10.1056/ NEJMoa1802357.

18. Dummer R, Brase JC, Garrett J, Campbell CD, Gasal E, Squires M, et al. Adjuvant dabrafenib plus trametinib versus placebo in patients with resected, BRAF(V600)-mutant, stage III melanoma (COMBI-AD): exploratory biomarker analyses from a randomised, phase 3 trial. Lancet On- 
col. 2020;21(3):358-72. https://doi.org/10.1016/S14702045(20)30062-0.

19. Amaria RN, Menzies AM, Burton EM, Scolyer RA, Tetzlaff MT, Antdbacka R, et al. Neoadjuvant systemic therapy in melanoma: recommendations of the International Neoadjuvant Melanoma Consortium. Lancet Oncol. 2019;20(7):e378-e89. https://doi.org/10.1016/S14702045(19)30332-8.

20. Amaria RN, Prieto PA, Tetzlaff MT, Reuben A, Andrews MC, Ross MI, et al. Neoadjuvant plus adjuvant dabrafenib and trametinib versus standard of care in patients with high-risk, surgically resectable melanoma: a single-centre, open-label, randomised, phase 2 trial. Lancet Oncol. 2018;19(2):181-93. https://doi.org/10.1016/S14702045(18)30015-9.

21. Amaria RN, Reddy SM, Tawbi HA, Davies MA, Ross MI, Glitza IC, et al. Neoadjuvant immune checkpoint blockade in high-risk resectable melanoma. Nat Med. 2018;24(11):1649-54. https://doi.org/10.1038/s41591018-0197-1.

22. Blank CU, Rozeman EA, Fanchi LF, Sikorska K, van de Wiel B, Kvistborg P, et al. Neoadjuvant versus adjuvant ipilimumab plus nivolumab in macroscopic stage III melanoma. Nat Med. 2018;24(11):1655-61. https:// doi. org/10.1038/s41591-018-0198-0.

23. Long GV, Saw RPM, Lo S, Nieweg OE, Shannon KF, Gonzalez $\mathrm{M}$, et al. Neoadjuvant dabrafenib combined with trametinib for resectable, stage IIIB-C, BRAF(V600) mutation-positive melanoma (NeoCombi): a single-arm, open-label, single-centre, phase 2 trial. Lancet Oncol. 2019;20(7):961-71. https://doi.org/10.1016/S14702045(19)30331-6.

24. Huang AC, Orlowski RJ, Xu X, Mick R, George SM, Yan PK, et al. A single dose of neoadjuvant PD-1 blockade predicts clinical outcomes in resectable melanoma. Nat Med. 2019;25(3):454-61. https://doi.org/10.1038/s41591-0190357-y.
25. Rozeman EA, Menzies AM, van Akkooi ACJ, Adhikari C, Bierman C, van de Wiel BA, et al. Identification of the optimal combination dosing schedule of neoadjuvant ipilimumab plus nivolumabin macroscopic stage IIImelanoma (OpACIN-neo): a multicentre, phase 2, randomised, controlled trial. Lancet Oncol. 2019;20(7):948-60. https://doi. org/10.1016/S1470-2045(19)30151-2.

26. Menzies AM, Rozeman EA, Amaria RN, Huang ACC, Scolyer RA, Tetzlaff MT, et al. Pathological response and survival with neoadjuvant therapy in melanoma: a pooled analysis from the International Neoadjuvant Melanoma Consortium (INMC). J Clin Oncol. 2019;37(15_suppl):9503. https://doi.org/10.1200/JCO.2019.37.15_suppl.9503.

27. Hindie E. What is the role of dabrafenib plus trametinib adjuvant therapy in stage IIIA melanoma? J Clin Oncol. 2019;37(15):1355-6. https://doi.org/10.1200/JCO.18. 02075.

28. Carlino MS, Ascierto PA, Eggermont AM, Gershenwald JE, Grob JJ, Hauschild A, et al. Pembrolizumab versus placebo as adjuvant therapy in resected high-risk stage II melanoma: Phase 3 KEYNOTE-716 study. J Clin Oncol. 2019;37(15_suppl):TPS9596. https://doi.org/10.1200/JCO. 2019.37.15_suppl.TPS9596.

Publisher's Note Springer Nature remains neutral with regard to jurisdictional claims in published maps and institutional affiliations.

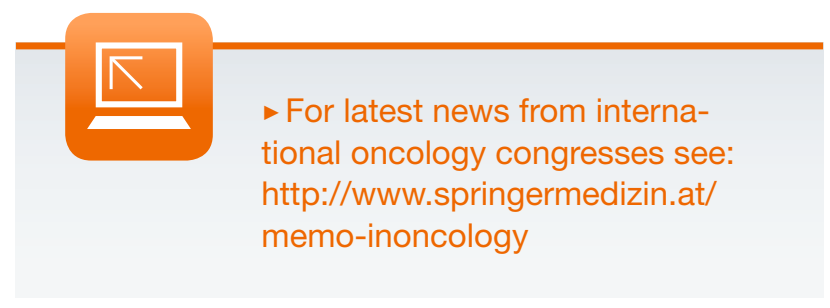

